Document downloaded from:

http://hdl.handle.net/10251/47986

This paper must be cited as:

Giménez Pérez, A.; Cibrián Ortíz De Anda, R.; Cerdá Jordá, S.; Girón, S.; Zamarreño García, T. (2014). Mismatches between objective parameters and measured perception assessment in room acoustics: a holistic approach. Building and Environment. 74:119-131. doi:10.1016/j.buildenv.2013.12.022.

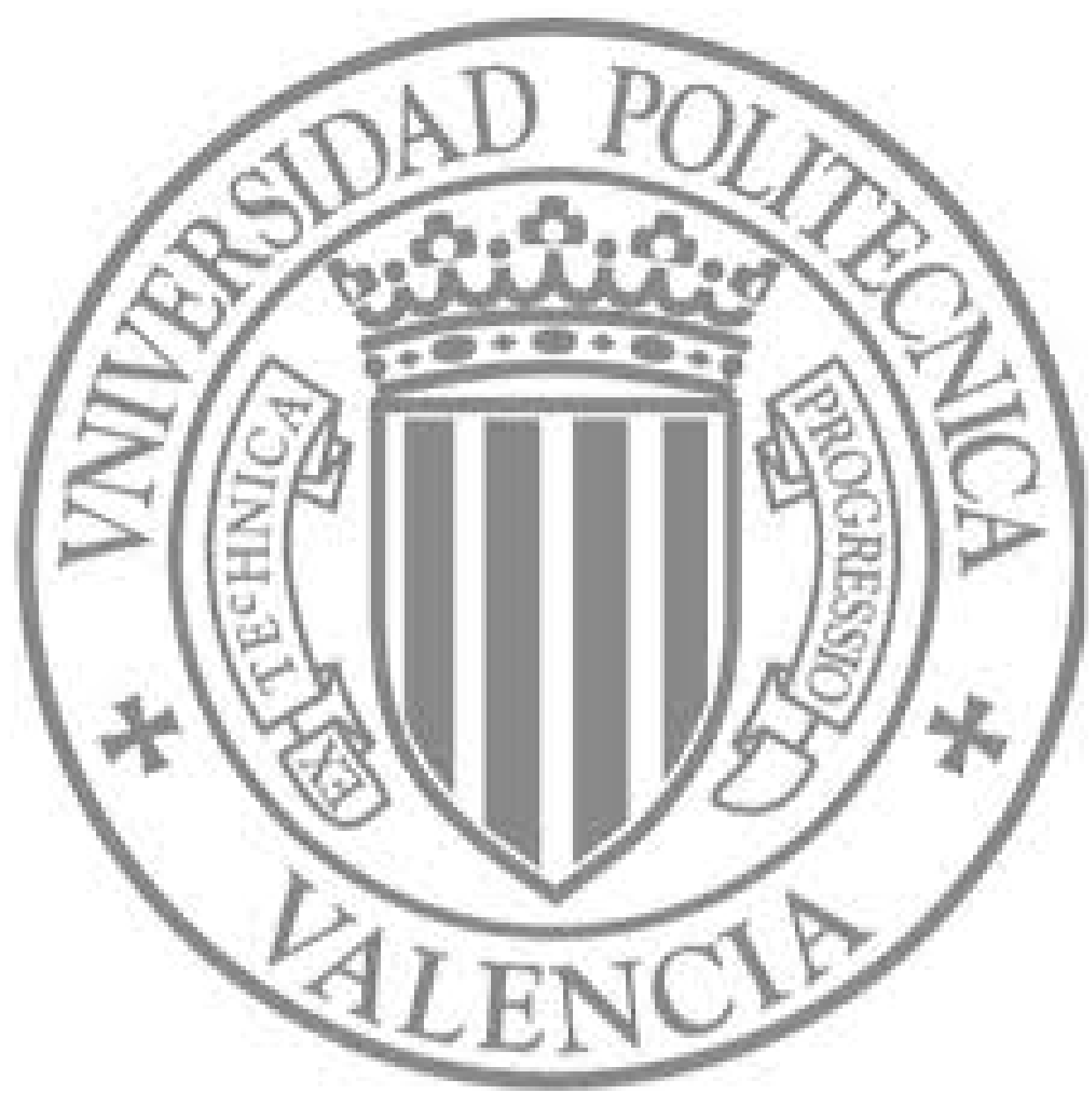

The final publication is available at

http://dx.doi.org/10.1016/j.buildenv.2013.12.022

Copyright Elsevier 


\section{Mismatches between objective parameters and measured perception assessment in room acoustics: a holistic approach}

Salvador Cerdáa, Rosa María Cibrián ${ }^{\mathrm{b}}$, Alicia Giménez ${ }^{\mathrm{a}}$, Sara Girón ${ }^{\mathrm{c}}$, Teófilo Zamarreño ${ }^{\mathrm{c}, *}$

${ }^{\mathrm{a}}$ Escuela Técnica Superior de Ingenieros Industriales, Universitat Politècnica de València

Camino de Vera s/n, 46022 Valencia, Spain; (e-mails: Salvasdor.Cerda@uv.es,

agimenez@fis.upv.es)

${ }^{\mathrm{b}}$ Facultad de Medicina, Universitat de València

Blasco Ibañez 15, Valencia, Spain; e-mail: Rosa.M.Cibrian@uv.es

${ }^{\complement}$ Escuela Técnica Superior de Arquitectura, IUACC, Universidad de Sevilla

Av. Reina Mercedes, 2, 41012 Seville, Spain. (e-mails: sgiron@us.es, teofilo@us.es)

*Corresponding author: teofilo@us.es, Tel. +34 954557129 / Fax: +34 954557892 


\begin{abstract}
Psychoacoustic research in the field of concert halls has revealed that many aspects concerning listening perception have yet to be totally understood. On the one hand, the objective room acoustics of performance spaces are reflected in parameters, some standardized and some not, but these are related to a limited number of perceptual attributes of human response. In general, these objective parameters cannot accurately describe the acoustic details due to their inherent simplification. Under these premises, impulse responses (576 receivers) are measured in 16 concert halls, according to standard procedures, and the perception and satisfaction of the occupants of the rooms are evaluated by completing a questionnaire during live concerts. Correlation analyses and multidimensional scaling (MDS) techniques have been applied to spatial and multi-band averaged values of the acoustic parameters studied (18), and the average values of users' responses (1284) to the questionnaire items (26). As a first result, correlations between objective parameters and users' responses show that transversality exists between them. Secondly, hierarchical clustering produces the classification of survey questions in 7 hierarchical classes. On the other hand, a lack of tuning between objective parameters and perceptual responses is observed on applying MDS analysis to the ordination of the venues from a subjective assessment and a subjective-objective assessment. Finally, although the results show the mismatch between objective parameters and subjective responses, a model of subjective global evaluation of the acoustics of the room from data of three orthogonal acoustic parameters is implemented, revealing a reasonably good fit.
\end{abstract}

Keywords: Perceptive acoustic evaluation, room acoustics, concert hall, multidimensional scaling, acoustic quality, concert-goers' responses. 


\section{Introduction}

Research in the subjective domain of the room acoustic experience is an essential aspect for intervention and design of halls for speech and music, and over the years a variety of techniques have been applied for the presentation of sound fields to individuals, all of which possess certain limitations. In the two basic methodologies of field measures (questionnaire or interview surveys for concert-goers, expert listeners or musicians) and laboratory experiments (presentations of recordings from halls or audible simulations to the listeners), the former lacks flexibility and control of the independent variables and the latter lacks fidelity. However, in both cases, multidimensional statistical analysis is required since it corresponds to data which involves listening experiments with test subjects.

Numerous listening experiments in real rooms and simulated sound fields over past years $[1,2]$ have established a certain number of perception attributes of sound fields and as a result of consensus they have been described in objective measures included in standards $[3,4]$. The diversity of acoustic design of a room and the complexity of human perception and acoustic information relating to a room justify the effort put into the research that analyzes correlations between objective measures (whether standardized or not) and the listening experiments of the acoustic comfort in concert venues. The consistency of the results in many studies validates this procedure for the investigation of the quality of the acoustic field in concert halls and the search for new, individually raised attributes [5]. By deliberately omitting certain pieces of research in the laboratory $[5,6]$ regarding concert halls and subjective studies of music perception in other architectural spaces $[7,8]$, the focus is centred on certain pieces of work from the two last decades, which, similar to this study, refer to live concerts. In this way, and in agreement with earlier studies using dummy-head recordings, from the results carried out in two concert halls, Sotiropoulou et 
al. $[9,10]$ identified, through correlation analysis, that the subjective factor body correlated with the low frequency $\mathrm{C}_{80}$ index, and that proximity correlated with the total-sound level and source-receiver distance. Cox et al. [11] also studied the acoustic quality of the Royal Festival Hall in London by using questionnaires. From the comparison of these responses with the measured objective parameters, they found, among other conclusions, that there was a correlation between subjective loudness, subjective liveliness and sound level, and that the balance between the soloist and the orchestra was correlated to $C_{80}$ and $T_{\mathrm{S}}$ parameters. In addition, in 23 opera houses from 11 countries, Hidaka et al. [12] measured room acoustical parameters: reverberation time $T$, early decay time EDT, clarity factor $C_{80}$, bass ratio BR, strength $G$, interaural cross-correlation coefficient IACC, and initial-timedelay gap ITDG under unoccupied conditions, which were analyzed for reliability and orthogonality. The experimental results were also related to the subjective responses of the questionnaires mailed to conductors in order to establish optimal ranges of the parameters; by executing multiple regression analysis, the authors demonstrate the importance of (1$\mathrm{IACC}_{\mathrm{E} 3}$ ) and ITDG for the approximation of the acoustical quality of opera houses. Likewise, Farina [13] researched the correlations between experimental measures (12 parameters) and subjective evaluations in order to find the acoustical parameters which are strongly related to the subjective judgments of acoustic comfort for listeners in opera houses in Italy. Statistical analysis by using multiple linear regressions between acoustic parameters and subjective pairs revealed very low coefficients of the correlations, and hence these results were used to create another, more refined questionnaire.

In this paper, the first method of subjective assessment is used, whereby the questionnaire is completed by the concert audience either during or shortly after the concert itself, in order to study the acoustic perception of a wide range of performance venues in the regions of Valencia and Andalusia, Spain, and their relationship with physical data by statistical 
methods. The process of depuration, refinement and statistical validation of the questionnaire used has already been published previously [14], based on the results obtained in various regions of the country, and where the different points of view of respondents [15] have also been assessed. Along this line, recent work has been carried out by Galiana et al. [16].

Multidimensional scaling (MDS) [17] encompasses a collection of methods which allow insight to be gained into the underlying structure of relations between entities by providing a geometrical representation of these relations. As such, these methods belong to the more general category of methods for multivariate data analysis. Multidimensional scaling can be characterised by the generality of the type of observed relations that can be submitted to the data analysis on the one hand, and by the specificity of the type of geometrical representation of these relations on the other hand. Any type of relation between a pair of entities that can either be translated into a proximity measure or, conversely, into a dissimilarity measure, can also be considered as possible input for multidimensional scaling. The choice of the particular type of spatial representation can be considered to be the most important part of the "modelling", and is applied together with a specific MDSalgorithm on the set of proximities. In the field of room acoustics, these techniques have been utilized in concert audition assessments [18], and more recently for a variety of purposes: to assess the perceptual similarity between auralizations, using both measured and simulated binaural room impulse responses [19]; for perceptions of double-slope decay profiles from auralized impulse responses of virtual hall configurations [20]; and for subjective evaluations with auralizations from on-site room impulse responses [21]. This paper presents four contributions to the field of acoustic studies of concert halls. First, 16 concert halls are measured (following ISO 3382-1 standard), and perceptively evaluated by using questionnaires in real concerts. Second, the correlations between mean values of 
objective parameters and mean values of the items of the questionnaires are studied. Third, the data analysis is further developed by including both the multidimensional scaling analysis (MDS) of questionnaires and the effects of objective parameters of the 16 concert halls surveyed. Finally, a preference-rating formula using objective data is developed. In line with the above, this paper is organized as follows. The procedures to measure objective parameters and the methodology of the subjective evaluation using surveys are reviewed first. The main results of the correlation analysis of subjective surveys and objective acoustic parameters are then shown. In addition, all data is analyzed in order to ascertain the links between objective and perception data. And finally, the preference rating is explained based on the objective data.

\section{Experimental procedures}

Measurements were carried out in two regions of Spain, one in an eastern community, Valencia, and the other in the south, Andalusia (see the map in Fig. 1), by two research teams: one from the Valencia Universities (The Polytechnic University and The General Study University) and the other from the University of Seville, who have participated in a joint research project [22] on acoustics of concert halls. The bulk of the experimental equipment is the same in both groups, showing only a few specific differences, as described below (Subsection 2.2). Additionally, before starting the measurement campaign, joint sessions of contrast between the two teams were conducted in Valencia. The methodology used in the surveys was also the same: a relatively unchanged group of experts for each region, consisting of music lovers, final-year students from the music conservatory, and music teachers, was placed in locations chosen in advance so that all parts of the seating area would be covered; the experts exchanged seats during the intermissions, and these seats coincided with the positions of the microphones for the 
objective acoustic measurements. Written questionnaires specially developed for this purpose [14] were completed during or immediately after hearing a live concert of symphonic music in the official programme of the concert hall. For concert-goers, the questionnaires, headed with an explanation of the aim of the research, were distributed at the entrance of the concert, and were collected at the exit.

\subsection{Researched halls}

The study was carried out in 16 theatres and auditoriums, from two Spanish autonomous communities: 8 halls in Valencia and 8 halls in Andalusia (see Fig.1). The most relevant data for these studied halls is shown in Fig. 2, alphabetically ordered according to the acronyms assigned to their Spanish names: Auditorio de Benaguacil (AB), Auditorio Manuel de Falla (AMF), Auditorio del Palacio de Congresos de Castellón (APC), Auditorio de Ribarroja (AR), L’Auditori de Torrent (AT), Gran Teatro de Córdoba (GTC), Gran Teatro Falla (GTF), Gran Teatro de Huelva (GTH), Palau de La Música (PAM), Paraninfo de la Universidad Politécnica de Valencia (PPV), Teatro Lope de Vega (TLV), Teatro de la Maestranza (TM), Teatro Miguel de Cervantes (TMC), Teatro Principal (TP), Teatro Unión Musical (TUM), and Teatro Villamarta (TV). In addition to showing an interior view of each hall, the acronym, name, location, year of construction or opening, typology, volume, number of seats, volume per seat, number of receptors R, and number of completed questionnaires Q, are also given in detail. All the music venues are available on the Mirem web site [23], except AB, APC and PPV halls. It should be mentioned that several halls have been refurbished, in particular their facilities, since the time the subjective measures were completed.

\subsection{Objective measurements}


In each hall, 18 objective parameters of acoustic quality descriptors are determined following the protocol of the ISO-3382-1 standard [3], and are spectrally averaged at each reception point, in accordance with said standard [3] and with the suggestions made by certain authors, as shown in Appendix A. The spatial averaging is achieved over the spectrally averaged values for each receiver position, and all measurements are accomplished in the unoccupied room and stage. Table 1 shows these mean values (single value) for each room, and Fig. 3 compares the variability of the results of the parameters in the halls. The data confirms that there is diversity in the halls studied in the sample. In this presentation, the acoustic parameters which assess speech intelligibility are deliberately omitted since the questionnaire refers to the perception of classical music in concert halls. During measurement, the environmental conditions are monitored by means of a precision electronic thermo-hygrometer, and a range of variation in Andalusian enclosures is revealed of 16.0 - $25.2{ }^{\circ} \mathrm{C}$ for the temperature, and 38 - $60 \%$ for the relative humidity, while in the Valencian halls these figures are 20.0 - $24.5^{\circ} \mathrm{C}$ for the temperature and 64 $70 \%$ for the relative humidity. The IRs are obtained at each reception point using sine sweep signals, running from 16 to $20,000 \mathrm{~Hz}$, which are generated and analyzed by the WinMLS 2004 software via the professional sound card VX Pocket v2 from Digigram. This generated signal feeds the INTER-M 1000 amplifier for its subsequent reproduction by the omnidirectional source AVM dodecahedral DO12 01-dB Stell loudspeaker, placed at $1.50 \mathrm{~m}$ from the floor and on the stage.

Various microphones are employed to attain the various parameters: G.R.A.S. Type 40 AK 1/2-inch and its corresponding supply source G.R.A.S. 12AA, and G.R.A.S Type 26AK preamplifiers are used in Valencia; while in Andalusia, omnidirectional B\&K Type 4190 1⁄2inch (with almost identical performance to G.R.A.S.) and B\&K Type 2669 pre-amplifiers with features similar to the cited $26 \mathrm{AK}$ are used, with appropriate signal-conditioning 
OPUS 01dB-Stell amplifiers. In order to obtain the directional parameters, an AudioTechnica AT4050/CM5 multi-pattern configurable microphone and the corresponding phantom supply source are used in the two regions. For the recording of binaural impulse responses, the torso-type HSU III simulator (Code 1323) from Head Acoustic is used in the two regions. All microphones are located at $\sim 1.20 \mathrm{~m}$ from the floor, in predetermined positions in the audience seating areas, covering all the audience area on their various levels. The number of receiver positions for the microphone in each hall is specified above the photograph of each performance space in Fig. 2.

\subsection{Subjective responses}

For the analysis of the response to the subjective acoustic experience, 1284 surveys, completed during or immediately after the concert by music experts (not in room acoustics) and the general public are considered. The whole survey consists of 58 questions (63 in the version for the orchestra musicians) grouped into 6 sections, which include both aspects of music perception (Sections A: General aspects; B: Detailed acoustic perception, and C: Overall acoustic perception), and sociological aspects (Sections D: Sociological and auditory data; E: Musical tastes; and F: Comments) [14]. For each room, the average values (Table 2) of the 26 questions of Sections B and C of the survey are obtained and shown in Appendix B. All items have the same rating scale from 0 to 5 points. In the averaged values, results from experts and concert-goers have been included (exclusively for responses in the audience area, and not for responses from musicians on stage), since a previous study of analysis and statistical validation of the survey concluded that these two types of answers can be used interchangeably [12]. Details of the audited concerts in each hall are shown in Appendix C; where two concerts were surveyed in the same hall, these are separated by a horizontal line. 
In Fig. 4, the averages of the responses to the questions are presented for each concert hall, and results show that the pattern is almost identically repeated in all performance places.

\section{Statistical results and discussion}

The aim of this work is to study the relationships between objective acoustic parameters measured in the room with the survey responses, which measure the quality of perceived acoustics of that room. Since objective parameters are used to physically characterize the acoustic quality of the room, with this study it can therefore be demonstrated which objective parameters and which survey questions are more relevant when performing a sound assessment of the rooms.

SPSS v19.0 software [24] was used for this study and various types of analysis were performed. As a first analysis, the correlations between the mean values of the items in each room with the averages of the objective parameters in each room were studied, as given in the sets of data from Tables 1 and 2. The Pearson correlation coefficient with bilateral significance test was used as an indicator. This type of analysis also appears in [13]. The application of techniques of hierarchical and multidimensional scaling (MDS) [17] to the same set of data of the halls provided the second type of analysis. Advantages of using MDS compared to other multivariate techniques include:

- Data can be measured on any scale, while in factor analysis, it must be measured as an interval or ratio.

- It provides solutions for each individual, which is impossible in factor analysis and in cluster analysis. This treatment enables the comparison of the assessments of each room since objective parameters and/or subjective assessments can be included. 
- The researcher does not need to specify the variables to use in the comparison of objects, which is central to factor analysis and cluster analysis, thus preventing the influence of the researcher in the analysis.

- Solutions provided by MDS are typically of smaller dimensionality than those provided by factor analysis.

- Distances between all points can be interpreted directly, whereas in correspondence analysis only distances between rows or between columns can be directly deduced. A hierarchical analysis of the survey items has primarily been performed, thus obtaining a geometric interpretation of the statistical associations between the averages of the responses. By repeating the analysis but this time including the objective parameters though MDS, the statistical effects can be seen geometrically and it can be found which group of objective parameters points in the same direction as the clusters of subjective parameters obtained previously. Finally, following a methodology similar to that developed in a previous study [25], a model of the degree of preference from orthogonal objective parameters is provided [26].

\subsection{Correlations between survey items and objective parameters}

Initially, the correlation between the mean objective parameters and the mean value of the answers of the questionnaire of the halls analyzed are assessed: data from Tables 1 and 2 . Those correlations with statistical significance are considered $(\mathrm{p}<0.05)$. However, within this group, only results for the values of the r-Pearson coefficient greater than or equal to 0.5 (in absolute value) are selected. This threshold is chosen since it would imply that at least a $25 \%$ variation of a variable would be influenced by the variation of the other variables. It can be seen that question C21 (rate the acoustics of the room overall) presents no correlations higher than this cut-off with any parameter, the highest correlations are 
with $\mathrm{Br}, \mathrm{EDT}_{\mathrm{mid}}$ and $T_{\mathrm{mid}}$, with values ranging between 0.43 and 0.47 . Given the importance of this issue, the two best correlations are considered even if they do not reach the cut-off of 0.5 (in absolute values).

In this regard, Fig. 5 depicts the set of correlations that satisfy the above criteria. This plotted graph shows that various parameters are correlated positively while others are correlated negatively with the survey items. From this representation, objective parameters were grouped into four categories with the criterion that the sign of the correlation coefficient is the same for all elements in the group, and that they have common acoustic features.

1. Spatiality: $J_{\mathrm{LF} 3}, J_{\mathrm{LF} 4}, J_{\mathrm{LFC} 4}, \mathrm{IACC}_{\mathrm{L} 3}$. Correlation is negative with survey items.

2. Clarity-Balance: $C_{80 \mathrm{av}}, C_{50 \mathrm{av}}, \mathrm{Br}, \mathrm{BR}$. Correlation is negative with almost all survey items.

3. Envelopment: $G_{\text {late }}, L_{\mathrm{J} 4}, \mathrm{LEV}$. Positive correlations are shown.

4. Reverberation: $T_{\text {mid }}, \mathrm{EDT}_{\mathrm{mid}}, T_{S 1 \mathrm{k}}$. Positive correlations are shown.

Three of the studied parameters, ITDG, $G_{125}$, and $G_{\text {mid }}$, show no correlation with any question of the questionnaire. In the case of spatiality, the observed average correlation coefficient is -0.63 . In the case of clarity-balance, this is -0.56 . For parameters that have shown positive correlations, the envelopment group has an average of 0.53 , while the group parameter of reverberation has an average correlation coefficient of 0.59 . These averages are representative because the clustering is performed taking into account the sign of the correlations.

Figure 5 shows the groups of objective parameters and the items with which they correlate. Likewise, the items of the questionnaire have been grouped according to the subjective aspects they value: impressions made of the concert, impressions related to the sound, and impressions about subjective size, spectral balance, clarity and overall perception. The 
transversality between the two groups of objective parameters and the subjective aspect evaluated can be noted perfectly. Hence it can be noted that spatiality parameters correlate with items included regarding impressions of the concert, impressions on subjective size, spectral balance, clarity and overall perception. Clarity parameters correlate with questions included in concert impressions, sound impression, impressions about subjective size, frequency balance, and overall perception. Paradoxically these parameters show no correlation with those questions whose aspects are directly related to subjectively evaluated clarity. On the other hand, objective parameters grouped under envelopment correlate with aspects related to concert impression, clarity and overall acoustic perception. Finally, parameters grouped under reverberation correlate with questions which include concert impression, sound impression, subjective size impression, and overall perception. It deserves mention that question $\mathrm{C} 21$, which asks the listeners to evaluate the acoustic quality of the hall as a whole, shows no significant correlation. Those which show correlation correspond largely to parameters of reverberation.

The above provides evidence of the complexity of room acoustics and of its future needs together with the defects observed, as also indicated in [21, 27]. Therefore, either a redefinition of the parameters (integration time, averaged over frequencies) is required, or new parameters that correlate specifically and that are orthogonal with subjective aspects (bi-orthogonality) must be found. One interesting approach is provided by the study of impulse responses from a viewpoint of time-frequency analysis proposed by Pätynen et al. [28]. On the other hand, every aspect that ISO 3382-1 specifies or omits to specify in the determination of acoustic parameters [29] should be checked, as should the effects of the type of excitation in the perception of the subjective characteristics [30].

\subsection{Multidimensional scaling I: survey items}


Multidimensional scaling (MDS) is a technique for the analysis of the similarity or dissimilarity of data on a set of objects. This technique attempts to model such data as distances between points in a geometric space. The main reason for doing this is that a graphical display of the structure of the data is desired; one that is much easier to understand than an array of numbers and, moreover, one that displays the essential information within the data, smoothing out noise [31]. Multidimensional scaling is used as a technique for data exploration and as a methodology for the discovery of psychological dimensions hidden within the data.

As a first approach to recognizing the hidden structures, hierarchical clustering [32] is used. Hierarchical clustering methods require no selection of the number of clusters to be searched and/or a starting configuration assignment. Instead, they require the user to specify a measure of dissimilarity between (disjoint) groups of observations, based on the pairwise dissimilarities between the observations in the two groups. As the name suggests, they produce hierarchical representations in which the clusters at each level of the hierarchy are created by merging clusters from the adjacent lower level. At the lowest level, each cluster contains a single observation. At the highest level, there is only one cluster containing all data. This type of graphical display is called a dendrogram. A dendrogram provides a highly interpretable complete description of the hierarchical clustering in a graphical format. This is one of the main reasons for the popularity of hierarchical clustering methods.

Thus, a first grouping was performed with agglomerative hierarchical clustering (AHC) based on Euclidean distances, i.e., each data vector starts in its own cluster, and pairs of clusters are merged as one moves up the hierarchy. The clustering is carried out in conjunction with Ward's minimum variance method, i.e., the squared Euclidean distance between data vectors. Figure 6 presents the dendrogram obtained. 
Table 3 shows in detail the questions in each group found in the hierarchical structure (dendrogram). It also includes the scores for each item in the two-dimensional space obtained by multidimensional scaling. These scores are taken into account when analyzing the obtained clusters. The first conclusion that can be drawn is that the structure of initial headings listed in the survey (Fig. 5 and Appendix B), is slightly modified into the following structure: HC1, Concert impression (scores in the first quadrant of the MDS); HC2, Sound impression (scores in the fourth quadrant in the MDS); HC3, Intimacy (scores in the second quadrant on the MDS); HC4, Bassiness (scores in the third quadrant in the MDS); HC5, Frequency balance (in this case the grouping obtained by AHC has remained. As positive and negative scores are in MDS, the length of the arrow shown in Fig. 7 is shorter than the others); HC6, Reverberance (third quadrant of MDS analysis); and HC7, Definition (given the singular item score, this has remained isolated).

Group HC1 is called Concert impression since it encompasses questions about the concert and includes the clear distinction of the soloist. On the other hand, the second group of items refers to the evaluation of sound and is therefore called Sound impression. The other grouped characteristics are associated to terms already used by other authors.

It is possible to compare these results with those obtained from other authors in preceding work. Hawkes and Douglas [18] found four to six individual factors in their studies that involved listening to real symphony orchestras in situ (Reverberance, Evenness (Balanced and Blend), Intimacy, Definition, Enjoyment and Brilliance). Soulodre and Bradley [33] found that preference correlated best with Clarity and Treble, but also to Loudness. Sotiropoulou et al. $[9,10]$ found that ordinary concert-goers describe their acoustical experiences with Body (full-bodied, full, voluminous), Clarity (clear, distinct), Tonal quality (of smooth tone, of rich tone), and Proximity (near, enveloping). And Lokki et al. [5, 21], found Clarity, Definition, Reverberance, Envelopment/Loudness, Bassiness, 
Proximity and a group named Undefined, by using agglomerative hierarchical clustering on elicited attributes.

The main innovation in our paper is that with well-established terms (Reverberance, Intimacy, Definition, Frequency balance, Bassiness), terms of a more generic nature are included, such as Sound Impression and Concert Impression. These latter terms are a combination of questions that use a more specific vocabulary. This concept is in accordance with the vast majority of authors, who consider that subjective assessments cannot be explained individually with one or several objective parameters and that studies of a more comprehensive nature are necessary in order to determine the relative importance of each of the subjective components of sound quality of concert halls and their related objective measures [27]. On the other hand, the perception of concert hall acoustics is complex and multidimensional by nature: individuals have different tastes and preferences, in such a way that the descriptions of perceptual attributes of halls can be ambiguous [5].

\subsection{Multidimensional scaling II: objective and survey items}

The clusters obtained in the previous section can also be presented geometrically by using the ALSCAL module from SPSS software [24]. ALSCAL is one of the current MDS modules in SPSS, and differs from other MDS programs in minimizing S-Stress rather than Stress, thereby fitting squared distances to squared dissimilarities. In both cases they are a measure of the fit, ranging from 1 (worst possible fit) to 0 (perfect fit). SPSS software provides both S-Stress and Stress [31]. The test is considered significant for Stress values lower than 0.2 .

In Fig. 7, each group of features is depicted in the two-dimensional space obtained by MDS. In order to study the relationships between the averages of the responses to the 
questions and of the objective parameters, the common factorial space is computed with all data to ascertain the relationships between subjective and objective parameters. Since the differences between variables with very high ratings may cancel out the differences between those variables with low scores, we opted for the usual process of normalization $\left(Z=\frac{X-\bar{X}}{\sigma}\right)$.

As shown in Fig. 8, in general, objective parameters are not aligned with features obtained in MDS from averages of the surveys. At a glance the following four ratings can be established by looking at each quadrant of the obtained two-dimensional space.

1. In the first quadrant, only objective parameters mainly related to clarity appear.

2. In the second quadrant, the objective parameters of spatiality and strength, and the subjective feature Bassiness appear.

3. Most of the subjective groups of characteristics appear in the third quadrant (Concert impression, Intimacy, Frequency balance, Reverberance and Definition, together with reverberation objective parameters ( $T_{\text {mid }}, \mathrm{EDT}_{\text {mid }}$ and $\left.T_{\mathrm{S} 1 \mathrm{k}}\right)$. This result is also obtained by Lokki et al. [21], in which objective parameters EDT $_{\text {mid }}$ and $C_{80 a v}$ at mid-frequencies are not perfectly aligned with subjective Reverberance and Definition (and Clarity) as suggested by ISO3382-1: 2009.

4. In the fourth quadrant, the Sound impression characteristic together with ITDG, IACC $_{\mathrm{E} 4}$ and BR parameters appear.

Multidimensional scaling analysis also enables the evaluation of rooms in the twodimensional space deduced from the variables. This analysis can be carried out by using the subjective assessments and adding the objective parameters. In order to compare the results, MDS analysis of the standardized variables is performed. Figure 9 shows the results of MDS analysis of the halls by using the subjective responses exclusively (red 
dots), and then by jointly analyzing subjective responses and objective parameters (blue squares). To better illustrate the effect of the inclusion of the objective parameters, a line connecting the various situations in the same room is drawn. In Fig. 9, this effect is clearly visible since the rooms reposition depending on whether they are represented on the MDS plane of subjective characteristics, or on the combined plane of subjective characteristics and objective parameters.

This effect can be interpreted to indicate that objective data does not match well with subjective data, since in this joint analysis the objective data pulls the data points in different directions to those of subjective data; in the same manner as occurs in [21]. However, it can be seen that, but for only three exceptions, halls always remain in the same quadrant (the most remarkable exception being that of the Maestranza Theatre (TM)). Hence we will use the interpretation of the quadrants that was done a little earlier to interpret Fig. 9.

First, it can be observed that there is a good variety of rooms. That is to say, subjective and objective characteristics (see Figs. 3 and 4) have sufficient variability to distinguish between rooms. In the first quadrant, i.e. the objective parameter of Clarity, there exist a large number of rooms (almost $40 \%$ of the total number of rooms). The other rooms are distributed equitably over the other three quadrants. Regarding the average of question C21 that serves as an overall assessment of the room, from the 8 best-valued, (in order: TM, AMF, TUM, AT, PAM, GTH, TV, and GTF), 6 are in the first quadrant. Only AT and GTH are not in the first quadrant, although in the former case, it is only when considering the hall subjectively. The case of GTH remains close to the origin.

As also shown in Fig. 9, several rooms suffer some notable variations. The greatest variation occurs in the case of the PPV room, followed by TM, TUM, TLV, APC, and PAM halls. This would indicate that the relationship between subjective responses and 
objective parameters is weaker in these rooms. When analyzing the overall assessment in these rooms, it can be observed that, by considering the three quality groups $\mathrm{A}, \mathrm{B}$ and $\mathrm{C}$ according to the average rating of question C21, the halls that suffer the largest deviations belong to these three groups. This fact can be interpreted to mean that, since those subjective factors that fail to match the objective parameters do not depend on the prestige of the room, therefore there is a real deficiency between objective parameters and subjective assessments.

On analyzing quadrant by quadrant, it can be observed that the halls of the first quadrant are differentiated by Concert impression when evaluated only subjectively, but they are differentiated by the objective parameters of clarity, $\mathrm{Br}, G_{125}$, and IACC $_{\mathrm{L} 3}$ when evaluated subjectively and objectively. In the second quadrant, subjective evaluation is differentiated by Intimacy, Frequency balance and Definition, and when evaluated jointly, by Bassiness and spatial and strength parameters. In the third quadrant, as for a purely subjective evaluation it is differentiated by Bassiness and Reverberance, but if objective parameters are included, then reverberation parameters and the majority of subjective characters (Intimacy, Definition, Frequency balance, Reverberance, Concert impression) appear. Finally in the fourth quadrant, by using MDS of subjective items, these are differentiated by Sound impression, and by also including objective parameters, then this feature remains together with $\mathrm{IACC}_{\mathrm{E} 3}, \mathrm{BR}$, and ITDG. Since changes of quadrants are unusual, these results can be interpreted as indicating that the subjective parameters that appear in the first analysis in each quadrant work similarly to those obtained in each quadrant of MDS, which are the combination of subjective assessment and of subjective-objective parameter assessment. It is concluded from this analysis, as in the correlation study carried out in Section 3.1, that although correlations between subjective assessments and objective parameters do exist, these correlations are not too high or, as shown in Fig. 8, subjective 
characteristics are not perfectly aligned with the objective parameters in the MDS analysis. This also follows the variation of the representation of the halls in the MDS space of subjective response when incorporating the objective parameters in the MDS analysis. This result is consistent with that of the work of Lokki et al. [21], in which a virtual orchestra is used as source [5] and the assessment is performed by an expert panel, whereby the procedure prescribed by ISO 3382-1 is not followed and the response to a real concert is not evaluated. It is concluded that although the benefits of using a virtual orchestra are undeniable since it is a source closer to reality, if the standard procedure is used with an omnidirectional source, a large number of measurements for averaging the objective parameters, and surveys to concert-goers, then the results are similar. Moreover, this method presents a significant advantage for the simplicity and repeatability of the procedure.

3.4 Model of subjective quality based on orthogonal objective parameters for halls for classical music

The most elaborate theory on the value of the subjective quality of a room from objective parameters is the "Theory of Subjective preference" devised by Ando [34]. The presentation of this theory by Beranek [35] provided the starting point for the development of the Ando-Beranek model, as laid out in [25]. This model was obtained by a linear combination of orthogonal objective parameters [26, 36]: $T_{\text {mid }}, J_{\mathrm{LFC} 4}$, and LEV. This combination correlates with that of Ando's functions $S_{i}$, which represents the scale value of a one-dimensional subjective response. The obtained combination is a new parameter, called $\mathrm{S}_{\mathrm{AB}}$, which enables the qualification of rooms and their quality ranking to be created:

$S_{A B}=-1.41+0.36 T_{\text {mid }}+2.76 J_{L F C 4}-0.19 L E V$ 
under the condition $-1 / 3<\mathrm{S}_{\mathrm{AB}}<0$ as a condition of good acoustic quality for a concert hall for classical music.

In the previous sections it has been observed that although there are correlations between the objective parameters and the subjective assessments, these are generally weak. Furthermore, no correlation appears between the average overall ratings by respondents and the objective parameters. Even though this can be interpreted as the result of a lack of new parameters or of modifications of existing parameters for better results [27], the methodological approach followed in [22] is maintained by seeking the best combination of orthogonal objective parameters [26, 36] that correlates with question C21, which is used to establish the ranking of halls. Although, in Eq. (1), $T_{\text {mid }}, J_{\mathrm{LFC} 4}$, and LEV [37] orthogonal parameters are used, in the first work on reducing parameters [26], it was observed that, given the correlation between BQI (Binaural quality index, BQI= (1IACC $_{\mathrm{E} 3}$ ) and $J_{\mathrm{LF}}$, a potentially successful selection of orthogonal parameters could be $T_{\text {mid }}, \mathrm{IACC}_{\mathrm{E} 3}$, and $\mathrm{LEV}$. This selection is more appropriate this time according to the result of MDS analysis with the groups of attributes and the objective parameters (Fig. 8), since these three objective parameters lie in the three quadrants that contain subjective characteristics. In order to proceed with the idea of achieving an explanation of the overall subjective satisfaction of a room through objective parameters, the corresponding line of linear regression is determined:

$$
C 21=3.909+0.585 T_{\text {mid }}-2.788 I{ }^{2} C_{E 3}-0.163 L E V \quad(r=0.7)
$$

This regression line is the solution to the problem of minimizing the distances of the data points to a linear model. However, as values of C21 are employed to establish a ranking of rooms, it would be advantageous to find a better combination of orthogonal acoustic parameters that provides a more similar arrangement. In order to determine whether two 
statistical variables show rank association, various coefficients can be calculated. The regression line leads to the definition of a parameter of sound quality (SQ) as follows:

$$
S Q=3.909+0.585 T_{\text {mid }}-2.788 I A C C_{E 3}-0.163 L E V
$$

By calculating the SQ parameter of each room, the Kendall-tau correlation [37] between C21 and SQ can be calculated. At this point, the variation of each SQ coefficient is proposed and the effect this variation has on the corresponding Kendall-tau correlation is observed.

Originally the Kendall-tau coefficient between C21 and SQ was 0.52. By means of the process of variation of parameters, the coefficient of Kendall-tau is now optimized with the expression:

$$
S Q K=4.2+0.58 T_{\text {mid }}-2.95 I A C C_{E 3}-0.12 L E V
$$

The new SQK parameter has a 0.6 Kendall-tau coefficient. This improvement entails a slightly smaller coefficient of linear correlation between C21 and SQK (0.67). In Fig. 10, C21, SQ, and SQK values for the halls ordered by the SQK parameter are shown. As can be appreciated in Fig. 10, the majority of rooms show an average response of overall assessment of the room, C21, which lies within the range determined by SQ and SQK. There are only two halls with C21 values that vary greatly from those obtained for SQ and SQK. These halls, APC and GTC, receive a very low evaluation in question C21. By analyzing the responses reached in the survey for these halls following a grouping by factors as achieved in [14], it can be seen that, in the APC case, there is a low score in factor F1: Pleasantness of the overall sound in the hall. In the case of the GTC hall, the low score is for factor F3: Overall perception of the orchestra. These facts are corroborated by comments from the technical staff of the halls, who, supported by general public opinion, estimate that some structural modifications are needed. However, objective parameters 
obtain good results with the proposed model, which indicates that sociological factors can affect the overall assessment of the halls.

This section can be concluded since, through multi-linear analysis and a method of optimization of Kendall-tau correlation, it has been possible to construct two parameters, SQ (Eq. 3), and SQK (Eq. 4), which enable the prediction of an interval of the subjective overall assessment of a hall from the $T_{\text {mid }}$, IACC $_{\mathrm{E} 3}$, and LEV parameters. This interval successfully works for all halls except in the case of two particular theatres whose overall assessment of acoustics is very low.

\section{Conclusions}

In this work, correlations are studied between subjective evaluations, obtained by means of questionnaires given to the general public and music experts during real concerts in various halls of Spanish territory, and averages of the most commonly used acoustic parameters. To this end, several statistical techniques are utilized. Firstly, the coefficients of the linear correlation between the average scores of the items in the questionnaires and objective parameters are calculated. It is deduced from this study that certain objective parameters correlate with various items, although, as a general rule, this correlation is weak. This has paved the way for the establishment of four groups of parameters that show correlations: Spatiality $\left(J_{\mathrm{LF} 3}, J_{\mathrm{LF} 4}, J_{\mathrm{LFC} 4}, \mathrm{IACC}_{\mathrm{L} 3}\right)$, Clarity-Balance $\left(C_{80 \mathrm{av}}, C_{50 \mathrm{av}}, \mathrm{Br}, \mathrm{BR}\right)$, Envelopment ( $\left.G_{\text {late }}, L_{\mathrm{J} 4}, \mathrm{LEV}\right)$, and Reverberation $\left(T_{\text {mid }}, \mathrm{EDT}_{\text {mid }}, T_{\mathrm{S} 1 \mathrm{k}}\right)$.

The two first groups show negative correlations with certain items, whilst those included in Envelopment and Reverberation show positive correlations. It should be borne in mind that the overall evaluation of a hall (question C21) shows no significant correlation with objective parameters. 
In the following sections, the agglomerate hierarchical technique and multi-dimensional scaling analysis are performed in order to relate subjective valuations and objective parameters. These statistical procedures have provided similar results to those attained by Lokki et al. [21] under different methodological conditions (virtual orchestra, a group of assessors, attributes elicited by the experts). The application of these methods to subjective evaluations have enabled the finding of 7 factors or clusters of subjective characteristics: HC1, Concert impression; HC2, Sound impression; HC3, Intimacy; HC4, Bassiness; HC5, Frequency Balance; HC6, Reverberance; and HC7, Definition. By carrying out the MDS analysis on the set of subjective responses and objective parameters it has been confirmed that, in general, objective parameters are not aligned with the subjective clusters of characteristics found. This fact, equivalent to low correlations between subjective evaluations and objective parameters, does not prevent the Reverberation parameters from being shown as those that present the greatest relationship with the majority of the subjective characteristics (HC1, Concert impression; HC2, Sound impression; HC3, Intimacy; HC4, Bassiness; HC5, Frequency Balance; HC6, Reverberance, and HC7, Definition). Furthermore, the Strength and Spatiality parameters show correlations with HC4, Bassiness; and IACC $_{\mathrm{E} 3}$, ITDG, and BR parameters show correlation with HC2, Sound Impression.

This lack of tuning between objective parameters and subjective assessments can also be appreciated on applying MDS analysis to the ordination of halls. When analyses are carried out with respect to subjective evaluations, and the analyses are repeated regarding joint subjective-objective evaluations, then halls suffer displacements within the bi-dimensional space defined by MDS. Nonetheless, as a general rule, these displacements maintain halls in the same quadrant. 
Finally, since the overall assessment of halls (question C21) fails to present any good correlation with any specific acoustic parameter, the methodology applied in [25] has been followed in order to obtain combinations of orthogonal objective parameters $[26,36]$ that correlate with question C21. Two parameters, SQ and SQK, are obtained which enable the definition of an interval within which the overall assessment of a hall is found. Therefore, objective parameters individually fail to describe the quality of the perceived sensation. This opens the way to investigate groups of parameters that describe said feeling, and to search for combinations of parameters that jointly enable closer resemblance to the perception received in the hall. 


\section{Acknowledgements}

The authors wish to express their gratitude to P. Bustamante for his help, to all those who participated as listeners in this study, and to management and staff of each hall for facilitating acoustic measurements and allowing distribution of the questionnaires in their theatres. This work has been financially supported by FEDER funds and by the Ministry of Science and Technology with references Nos. BIA2003-09306, BIA2008-05485, BIA 2010-20523, and BIA 2012-36896. 
Appendix A.

Acoustic parameter used in this study. Spectral average and reference.

\begin{tabular}{|c|c|c|}
\hline Parameter & Equation & Ref. \\
\hline Reverberation time & $T_{\text {mid }}=\frac{1}{2}\left(T^{500 \mathrm{~Hz}}+T^{1 k \mathrm{~Hz}}\right)$ & {$[3]$} \\
\hline Early decay time & $E D T_{\text {mid }}=\frac{1}{2}\left(E D T^{500 \mathrm{~Hz}}+E D T^{1 k \mathrm{~Hz}}\right)$ & {$[3]$} \\
\hline Brilliance & $\mathrm{Br}=\frac{T^{2 k \mathrm{~Hz}}+T^{4 k \mathrm{~Hz}}}{T^{500 \mathrm{~Hz}}+T^{1 k \mathrm{~Hz}}}$ & [38] \\
\hline Bass ratio & $B R=\frac{T^{125 \mathrm{~Hz}}+T^{250 \mathrm{~Hz}}}{T^{500 \mathrm{~Hz}}+T^{1 \mathrm{kzz}}}$ & [38] \\
\hline Initial time delay gap & ITDG & {$[38]$} \\
\hline Speech clarity & $C_{50 a v}=\left(0.15 C_{50}^{500 \mathrm{~Hz}}+0.25 C_{50}^{1 \mathrm{kHz}}+0.35 C_{50}^{2 \mathrm{kHz}}+0.25 C_{50}^{4 \mathrm{kHz}}\right)$ & {$[39]$} \\
\hline Clarity & $C_{80 a v}=\frac{1}{3}\left(C_{80}^{500 \mathrm{~Hz}}+C_{80}^{1 k \mathrm{~Hz}}+C_{80}^{2 k \mathrm{~Hz}}\right)$ & [39] \\
\hline Centre time & $T_{S 1 k}$ & {$[40]$} \\
\hline $\begin{array}{l}\text { Early and Late Interaural } \\
\text { cross-correlation } \\
\text { coefficients }\end{array}$ & $\left.\begin{array}{l}I A C C_{E 3}=\frac{1}{3}\left(I A C C_{E}^{500 \mathrm{~Hz}}+I A C C_{E}^{1 k \mathrm{~Hz}}+I A C C_{E}^{2 k H z}\right) \\
I A C C_{L 3}=\frac{1}{3}\left(I A C C_{L}^{500 \mathrm{~Hz}}+I A C C_{L}^{1 k H z}+I A C C_{L}^{2 k H z}\right)\end{array}\right\}$ & {$[41]$} \\
\hline Sound strength & $\begin{array}{l}G_{125} \\
G_{\text {mid }}=\frac{1}{2}\left(G^{500 H z}+G^{1 k H z}\right)\end{array}$ & {$[38]$} \\
\hline Late sound strength & $G_{\text {late }}=G_{\text {mid }}-10 \log \left(1+10^{C_{80 A V} / 10}\right)$ & {$[38]$} \\
\hline Late envelopment & $L E V=0.5 G_{\text {late }}+10 \log \left(1-I A C C_{L 3}\right)$ & {$[38]$} \\
\hline Late lateral sound level & $\begin{array}{l}L_{J 4}=\frac{1}{4}\left(L_{J}^{125 \mathrm{~Hz}}+L_{J}^{250 \mathrm{~Hz}}+L_{J}^{500 \mathrm{~Hz}}+L_{J}^{1 k \mathrm{~Hz}}\right) \\
J_{L F 3}=\frac{1}{3}\left(J_{L F}^{500 \mathrm{~Hz}}+J_{L F}^{1 k \mathrm{~Hz}}+J_{L F}^{2 k \mathrm{~Hz}}\right)\end{array}$ & $\begin{array}{l}{[42]} \\
{[38]}\end{array}$ \\
\hline $\begin{array}{l}\text { Early lateral energy } \\
\text { fraction }\end{array}$ & $\left.\begin{array}{l}J_{L F 4}=\frac{1}{4}\left(J_{L F}^{125 \mathrm{~Hz}}+J_{L F}^{250 \mathrm{~Hz}}+J_{L F}^{500 \mathrm{~Hz}}+J_{L F}^{1 k \mathrm{~Hz}}\right) \\
J_{L F C 4}=\frac{1}{4}\left(J_{L F C}^{125 \mathrm{~Hz}}+J_{L F C}^{250 \mathrm{~Hz}}+J_{L F C}^{500 \mathrm{~Hz}}+J_{L F C}^{1 k \mathrm{~Hz}}\right)\end{array}\right\}$ & {$[3]$} \\
\hline
\end{tabular}


Appendix B.

Reproduction of the 26 questions of the questionnaire used in this work.

Code Question

B01 Can you clearly distinguish the sounds of the different instruments?

B11 Can you clearly distinguish the soloist (if there is one)?

B21 How do you perceive the orchestra overall?

I consider that in this hall:

B41 The high sounds predominate (high frequencies)

B42 The low sounds predominate (low frequencies)

B43 The sound is perceived equally from all directions

Having heard the music, the sensation that it produces with respect to the proximity to or distance from the musicians is that of a hall that is...

B51 Large

B52 Open

B53 Wide

You find the sound of the hall:

B61 Reverberating (persistence of the sound, the different sounds are superimposed)

B62 Dry (the sounds are not prolonged sufficiently)

B63 Intimate (the music gives the impression of being played in a small enclosure)

B64 Lively, rich, brilliant (richness of high tones and slowness of their disappearance)

B65 Warm (it is rich in low sounds and they are perceived and distinguished clearly)

B66 Clear (the details of the musical execution are distinguished separately)

B67

Blurred (the individualized sounds of the musical execution are confused and mixed

The concert heard in this hall produces the sensation that the music is...

B71 Smooth, the contrary would be rough

B72

Exciting (predominance of high sounds and a loud level), the contrary would be calm

B73 Balanced, the contrary would be distorted

B74 Loud (loud sound), the contrary would be weak

B75 Pleasant, the contrary would be unpleasant

B76 Light, the contrary would be dense

B81 Do you feel enveloped by the sound?

C01 How loud do you perceive the orchestra overall?

C02 How balanced do you perceive the orchestra overall?

C21 How do you classify the acoustics of this hall overall? 
Appendix C.

Concerts performed in the halls.

\begin{tabular}{|c|c|c|c|}
\hline $\begin{array}{l}\text { AB } \\
\text { L. Bernstein (Divertimento } \\
\text { for orchestra); } \\
\text { F. Miller (The Forest of } \\
\text { Tears); } \\
\text { N. Rimsky-Korsakov } \\
\text { (Scheherazade). } \\
\text { M. Gálvez-Taroncher } \\
\text { (Night of Sobs); } \\
\text { M. Ohana (Concert for } \\
\text { Piano and Orchestra): } \\
\text { I. Stravinsky (The Firebird }\end{array}$ & $\begin{array}{l}\text { AMF } \\
\text { W. A. Mozart (Overture Le } \\
\text { Nozze di Figaro), } \\
\text { (Concierto for Piano and } \\
\text { Orchestra in C Major, No. } \\
\text { 21, K 467); } \\
\text { D. Shostakovich } \\
\text { (Concierto for Piano, } \\
\text { Trumpet and String } \\
\text { Orchestra, No. } 1 \text { in C } \\
\text { Minor, Op 35), (Ballet } \\
\text { Suite, No. } 3 \text { Op 91d). }\end{array}$ & $\begin{array}{l}\text { APC } \\
\text { M. Ravel (The Gracioso's } \\
\text { Aubade), (Tzigane); } \\
\text { C. Saint-Saëns (Havanera } \\
\text { Op 83); } \\
\text { I. Albéniz (Five Pieces of } \\
\text { Iberia). }\end{array}$ & $\begin{array}{l}\text { AR } \\
W . \text { A. Mozart (The } \\
\text { Marriage of Figaro) } \\
\text { C.M. von Weber (Der } \\
\text { Freiscutz); R. Strauss (Don } \\
\text { Juan); } \\
\text { J. Brahms (Symphony No. } \\
2 \text { in D Major). - }\end{array}$ \\
\hline $\begin{array}{l}\text { AT } \\
\text { G. Verdi (Rigoletto). } \\
\text { E. Bermell (Dolores } \\
\text { Tormo); L. Bernstein } \\
\text { (Divertimento for } \\
\text { orchestra); } \\
\text { G. Gershwin (Rhapsody in } \\
\text { Blue); } \\
\text { F. A. Comos (Carmina } \\
\text { Gueguel Massmanian } \\
\text { Estelles); } \\
\text { J. G. Gómez-Deval (O } \\
\text { Camino de Santiago); } \\
\text { M. Gould (Jericho). }\end{array}$ & $\begin{array}{l}\text { GTC } \\
\text { J. Brahms (Piano Concierto } \\
\text { No. 1); } \\
\text { H. Villa Lobos (Bachianas } \\
\text { Brasileiras No. 2); I. } \\
\text { Stranvinsky (The Firebird). }\end{array}$ & $\begin{array}{l}\text { GTF } \\
\text { M. Ravel (Pavane for a } \\
\text { Dead Princess); } \\
\text { J. Ibert (Four Songs of Don } \\
\text { Quixote); } \\
\text { G. F. Handel (Water } \\
\text { Music, Suite in F, HWV } \\
\text { 348). }\end{array}$ & $\begin{array}{l}\text { GTH } \\
\text { E. Hoyo, A. Step Díaz and } \\
\text { S. Aramburu: Libretto } \\
\text { authors (The Kiss Legend), } \\
\text { Music: R. Soutullo and J. } \\
\text { Vert. }\end{array}$ \\
\hline
\end{tabular}

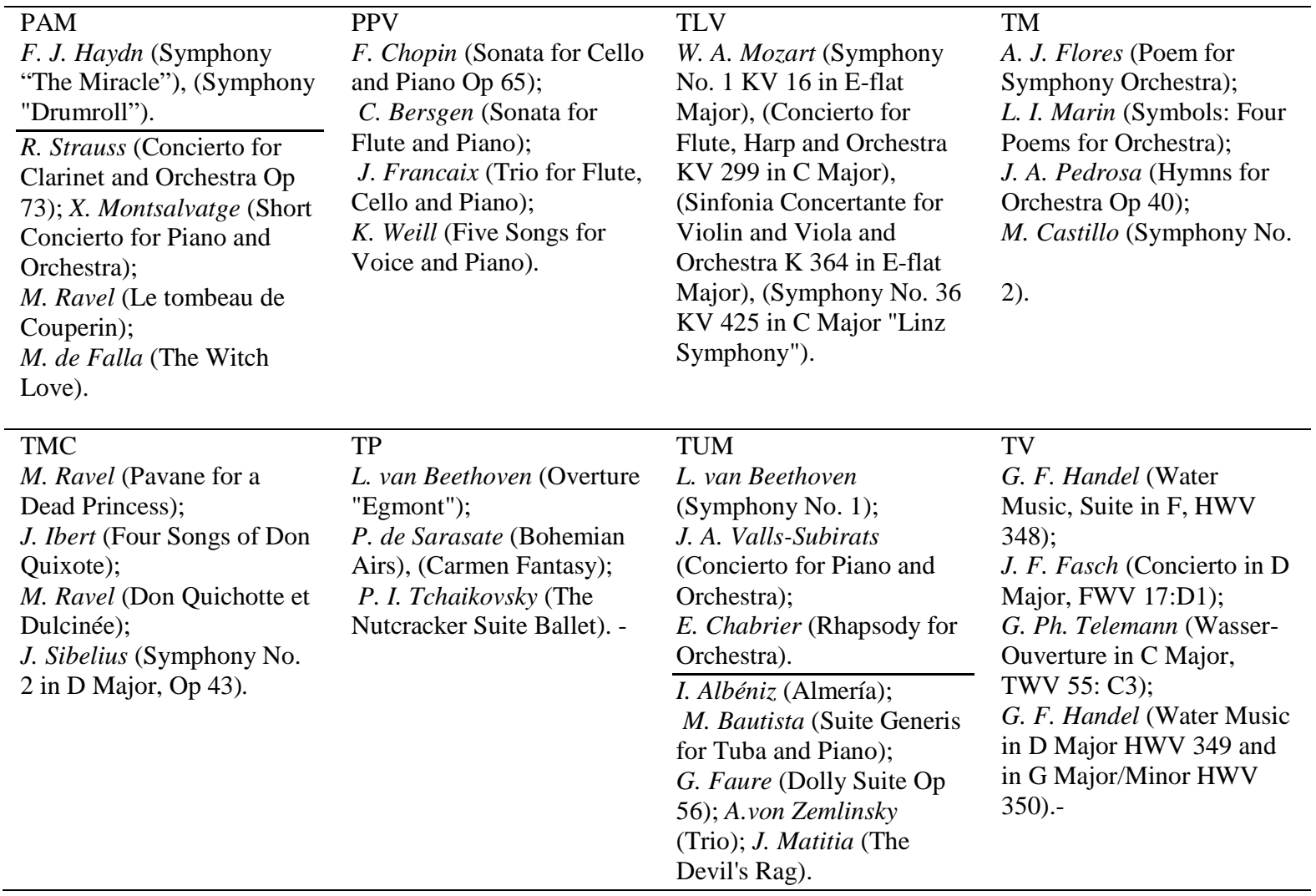




\section{References}

[1] Schroeder MR, Gottlob D. Siebrasse F. Comparative study of European concert halls-correlation of subjective preference with geometric and acoustic parameters. $\mathrm{J}$ Acout Soc Am 1974;56:1195-1201.

[2] Oldham DJ, Rowell MA. Computer applications in building and environmental acoustics. Build Environ 1987;22:189-200.

[3] International Standard ISO 3382-1:2009(E) Acoustics - Measurement of room acoustic parameters. Performance spaces, International Organization for Standardization, Geneva, Switzerland, 2009.

[4] IEC 60268-16. Sound system equipment, Part 16: Objective rating of speech intelligibility by speech transmission index. International Electrotechnical Commission. Geneva, Switzerland, 2003.

[5] Lokki T, Pätynen J, Kuusinen A, Vertanen H, Tervo S. Concert hall acoustic assessment with individually elicited attributes. J Acout Soc Am 2011;130:835-49.

[6] Sato S, Prodi N. On the Subjective evaluation of the perceived balance between a singer and a piano inside different theatres. Acta Acustica united Acustica 2009;95:519-26.

[7] Carvalho APO, Morgado AEJ, Henrique L. Relationship between subjective and objective acoustical measures in churches. Build Acoust 1997;4:1-20.

[8] Martellotta F. Subjective study of preferred listening conditions in Italian Catholic churches. J Sound Vib 2008;317: 378-99.

[9] Sotiropoulou AG, Hawkes RJ, Fleming DB. Concert hall acoustic evaluations by ordinary concert-goers: I. Multidimensional description of the evaluations. Acustica 1995;81: 1-9. 
[10] Sotiropoulou, AG, Fleming DB. Concert hall acoustic evaluations by ordinary concert-goers: II. Physical room acoustic criteria subjectively significant. Acustica 1995;81;10-9.

[11] Cox TJ, Shield BM: Audience questionnaire survey of the acoustics of the Royal Festival Hall, London, England. Acustica 1999;85:547-59.

[12] Hidaka T, Beranek LL. Objective and subjective evaluations of twenty-three opera houses in Europe, Japan, and the Americas. J Acoust Soc Am 2000;10;368-83.

[13] Farina A. Acoustic quality of theatres: correlations between experimental measures and subjective evaluations. Appl Acoust 2001;62: 889-916.

[14] Giménez A, Cibrián RM, Girón S, Zamarreño T, Sendra JJ, Vela A, Daumal F. Questionnaire survey to qualify the acoustics of Spanish concert halls. Acta Acustica united Acustica 2011;97: 949-65.

[15] Gimenez A, Cibrian RM, Cerdá S. Subjective assessment of concert halls: a common vocabulary for music lovers and acousticians. Arch Acoust 2012;37:331-40.

[16] Galiana M, Llinares C, Page A. Subjective evaluation of music hall acoustics: Response of expert and non-expert users. Build Environ 2012;58:1-13.

[17] Cox TF, Cox MAA. Multidimensional scaling. 2nd ed. London: Chapman \& Hall; 2001.

[18] Hawkes RJ, Douglas H. Subjective acoustics experience in concert auditoria. Acustica 1971;24:235-50.

[19] Zahorik P. Perceptually relevant parameters for virtual listening simulation of a small room acoustics. J Acoust Soc Am 2009;126:776-91.

[20] Bradley DT, Wang LM. Optimum absorption and aperture parameters for realistic coupled volume spaces determined from computational analysis and subjective testing results. J Acoust Soc Am 2010;127:223-32. 
[21] Lokki T, Pätynen J, Kuusinen A, Tervo A. Disentangling preference ratings of concert hall acoustics using subjective sensory profiles. J Acoust Soc Am 2012;132:3148-61.

[22] URL: http://grupo.us.es/gteacus/ (visited 16/12/2013).

[23] MIREM (Mapa Informatizado de Recintos Escénicos y Musicales; Fundación Autor, Sociedad General de Autores y Editores) http://www.mirem.net/ [Digital Scenic and Musical Enclosure Map, Author Foundation] (visited 16/12/2013, currently only in Spanish).

[24] IBM SPSS Statistics 19 Core System,User’s Guide Licensed Materials - Property of SPSS Inc., an IBM Company. ㄷ Copyright SPSS Inc. 1989, 2010. Patent No. $7,023,453$.

[25] Cerdá S, Giménez A, Cibrián RM. An objective scheme for ranking halls and obtaining criteria for improvements and design. J Audio Eng Soc 2012;60:419-30.

[26] Cerdá S, Giménez A, Romero J, Cibrián RM, Miralles JL. Room acoustical parameters: a factor analysis approach. Appl Acoust 2009;70:97-109.

[27] Bradley JS. Review of objective room acoustics measures and future needs. Appl Acoust 2011;72:713-20.

[28] Pätynen J, Tervo S, Lokki T. Analysis of concert hall acoustics via visualizations of time-frequency and spatiotemporal responses. J Acoust Soc Am 2013;133:842-57.

[29] Hak CCJM, Wenmaekers RHC, Van Luxemburg LCJ. Measuring room impulse responses: impact of the decay range on derived room acoustic parameters. Acta Acustica united Acustica 2012;98:907-15.

[30] Billon A, Embrechts JJ. Discrimination thresholds of the reverberation in large volumes by naïve listeners. In: Proceedings of Acoustics 2012 Conference. 23-27 April 2012, Nantes, France. 
[31] Borg I, Groenen PJF. Modern multidimensional scaling. Theory and applications. 2nd ed. New York: Springer Science+Business Media Inc; 2005.

[32] Hastie T, Tibshirani R, Friedman J. The elements of statistical learning. data mining, inference, and prediction. 2nd ed. Springer-Verlag; 2008.

[33] Soulodre G, Bradley J. Subjective evaluation of new room acoustic measures. J Acoust Soc Am 1995;98:294-301.

[34] Ando Y. Concert hall acoustics. 1st ed. Berlin: Springer-Verlag; 1985.

[35] Beranek LL. Concert and opera halls. How they sound. 1st ed. New York: Acoustical Society of America; 1996.

[36] Cerdá S, Giménez A, Romero J, Cibrián RM. A factor analysis approach to determining a small number of parameters for characterising halls. Acta Acustica united Acustica 2011;97:441-52.

[37] Nelsen RB (originator). Kendall tau metric. Encyclopedia of Mathematics. URL: http://www.encyclopediaofmath.org/index.php?title=Kendall_tau_metric\&oldid=128 69 (visited 16/12/2013).

[38] Beranek LL. Concert Hall Acoustics-2008. J Audio Eng Soc 2008;56: 532-44.

[39] Marshall LG. An acoustic measurement program for evaluating auditoriums based on the early/late sound energy ratio. J Acoust Soc Am 1994;96:2251-61.

[40] Hoffmeier J. Untersuchungen zum Einfluß von Raumklang- färbungen auf die Deutlichkeit von Sprache (Investigations on the influence of room timbres on speech definition, in German) Technical University of Denmark Thesis for a degree at the TU Dresden, 1996. Referenced in Ahnert W, Tennehardt HP Acoustics for Auditoriums and concert halls. In: Ballou GM, editor. Handbook for Sound Engineers. Amsterdam: Elsevier; 2005, p. 109-55. 
[41] Okano T, Beranek LL, Hidaka T. Relations among interaural cross-correlation coefficient (IACCE), lateral fraction (LFE), and apparent source width (ASW) in concert halls. J Acoust Soc Am 1998;104:255-65.

[42] Barron M. Late lateral energy fractions and the envelopment question in concert halls. Appl Acoust 2001;62:185-202. 


\section{Figure captions}

Fig. 1. Location of the concert halls in Spain.

Fig. 2 Interior view of the performance spaces and relevant data: acronym, name, location, year of construction or opening, typology, volume, number of seats, volume per seat, number of receptors R, and number of completed questionnaires Q. Data for all venues is collected without an orchestra shell on the stage, except for GTC, GTF, TM, TMC and VT.

Fig. 3. Variability of the objective parameters studied in the different concert halls.

Fig. 4. Averages of the responses to the questions of the questionnaires in each concert hall.

Fig. 5. Graphical representation of the correlations between the survey questions, grouped according to the subjective attribute they value, and the objective parameters, grouped under spatiality, clarity-balance, reverberation and envelopment.

Fig. 6. Dendrogram obtained by hierarchical clustering using Ward’s minimum variance method.

Fig. 7. MDS with average vectors of attribute groups.

Fig. 8. MDS with average vectors of attribute groups and objective parameters.

Fig. 9. Ordination of concert halls in common bi-dimensional space.

Fig. 10. C21, SQ and SQK versus rank-ordination of concert halls by SQK. 